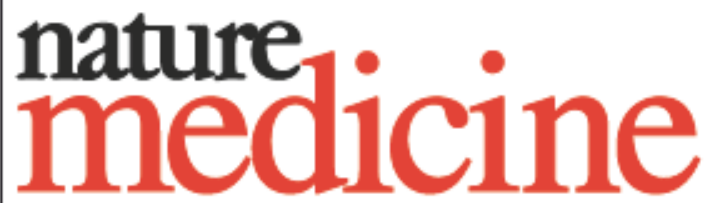

VOLUME 7 - NUMBER 4 - APRIL 2001

\title{
Prospects for Parkinson disease
}

The first randomized double-blind placebo-controlled trial of fetal transplantation for Parkinson disease published in the 8 March issue of $\mathrm{New}$ England Journal of Medicine has been the subject of much scrutiny by the popular press. The press characterized the NIHsupported study as a major setback for Parkinson research, but a more levelheaded analysis shows that in fact this study offers much hope for Parkinson patients.

Curt Freed and his colleagues at the University of Colorado and at Columbia University in New York employed a cohort of $\mathbf{4 0}$ patients, half of whom received dopaminergic implants each derived from four human fetal tissue fragments maintained in vitro for up to one month, the other half of whom went through a sham procedure which included drilling of holes in the skull, but no injection. For evaluation at one year after surgery or sham procedure, the investigators chose an unusual primary outcome parameter, a subjective measure wherein each patient evaluated his or her own condition. The patients' selfevaluations were highly variable and did not show a statistically significant effect of the transplantation, and this was the conclusion seized upon and highlighted by the press. But more conventional objective parameters of the progression of Parkinson disease show that the transplantation resulted in significant improvements, especially for patients under the age of 60 . Improvement occurred in signs of rigidity and, in the younger patients, sluggishness. As for the negative side-effects (dyskinesias) observed in $15 \%$ of transplanted patients at one to three years after surgery, the authors attributed this to "overdose" of tissue, and plan further studies with less tissue, more precisely placed within the putamen.
In just a few months, another group of researchers will begin to analyze the results of their own NIH-funded study of fetal tissue transplantation. Headed by Warren Olanow at Mount Sinai Medical Center in New York, it too is a randomized, placebo-controlled double-blind study of fetal tissue transplantation for severe Parkinson disease. But their study differs in several technical aspects. All patients receive immunosuppressive therapy which might contribute to better graft tolerance. Like the Freed study, these investigators are using solid fetal tissue grafts at four per patient, but the tissue has been stored in vitro for no longer than $\mathbf{4 8}$ hours. Olanow's surgical approach targets a more restricted area which he believes will result in a greater concentration of transplanted cells. Patients will be followed in a doubleblind manner for two years, instead of one year, and investigators will use objective measurements of improvement, not subjective self-assessments.

The historical precedent for these studles was the partial success of a number of transplantation studies in animal models and in small, uncontrolled studies on patients at various centers in Europe, Canada, and the US. These promising results and the urgent need for better therapies combined with the Clinton administration's lifting of the ban on fetal tissue research allowed the NIH to fund controlled trials. However, interpretation of these studies is complicated by the lack of optimization and standardization of the cells and procedure. Most clinical and basic scientists involved in neural transplantation agree that the field is in its infancy and that optimal methods of tissue preparation and implantation are not yet established. The optimal surgical approach also has not been worked out and surgeons differ widely in what they consider to be the best approach. Moreover, previous studies indicate that long-term patient follow-up is critical.

Clearly, with the use of fetal tissue that is not easily standardized and the need for as many as four embryos per patient, the future of transplantation therapy lies in the development of alternative sources of cells. Much work has been done over the past few years to generate dopaminergic neurons from in vitro expanded progenitors cells from the rat midbrain, but transplantation studies in rats indicate that different types of dopaminergic neurons vary greatly in their capacity to function in vivo, and that nondopaminergic cells in the graft may also contribute to engraftment. Multipotent stem cells might become an unlimited and self-renewing source for transplantation, but we need to learn much more about the basic biology of stem and progenitor cells before they can be tested in humans. Along these lines, the recent decision by the British government to approve the creation and use of human embryos for such research (see page 396 of this issue) is welcome. Although the Bush administraton has yet to set its policy on human fetal tissue/stem cell research, they would do well to follow the example set by their British counterparts.

Given the tremendous implications for further funding and research, care in study design and interpretation are of utmost importance. Considering the preliminary stage of transplant design and the one-year evaluation endpoint, the small improvements achieved by this first controlled study are remarkable and should encourage further research. Strong support for research that emphasizes collaboration among basic and clinical scientists is clearly necessary before the full benefits of neural cell transplantation can be realized. 